International Journal of Pure and Applied Mathematics

Volume 104 No. 1 2015, 145-149

ISSN: 1311-8080 (printed version); ISSN: 1314-3395 (on-line version)

url: http://www.ijpam.eu

doi: http://dx.doi.org/10.12732/ijpam.v104i1.12

\title{
THE SQUARE OF DIRAC OPERATOR ON HOMOGENEOUS SPACES
}

\author{
Liangzhong $\mathrm{Hu}$ \\ Department of Mathematics \\ Federal University of Paraná \\ C.P. 019081, Curitiba, PR, 81531-990, BRAZIL
}

\begin{abstract}
The solution of the eigenvalue problem of the square of Dirac operator with the normal metric induced by the Killing form on a general homogeneous space $G / H$ is given.
\end{abstract}

AMS Subject Classification: 22E65, 51P05, 81R10

Key Words: Dirac operator, highest weight, homogeneous space, Laplacian, Weyl group

\section{Introduction}

In [1], we gave the solution of the eigenvalue problem of the Laplacian on a homogeneous space $G / H$, where $G$ is a compact, semi-simple Lie group, $H$ is a closed subgroup of $G$, and the rank of $H$ is equal to the rank of $G$.

In this short paper, we give the solution of the eigenvalue problem of the square of Dirac operator with the normal metric induced by the Killing form on the same homogeneous space $G / H$.

Received: August 28, 2015

(c) 2015 Academic Publications, Ltd. url: www.acadpubl.eu 


\section{The Square of Dirac Operator on $G / H$}

We first review the Laplacian on a homogeneous space $G / H$. Here $G$ is a compact, semi-simple Lie group, $H$ is a closed subgroup of $G$, and the rank of $H$ is equal to the rank of $G$.

Let $\mathbf{g}$ and $\boldsymbol{\eta}$ be the Lie algebras of $G$ and $H$, respectively.

We suppose that $G / H$ is reductive, i.e. $\mathrm{g}$ has an orthognal decomposition $\mathbf{g}=\boldsymbol{\eta} \oplus \mathbf{m}$ with $[\boldsymbol{\eta}, \mathbf{m}] \subset \mathbf{m}$ and $[\mathbf{m}, \mathbf{m}] \subset \mathbf{g}$.

We can choose a common Cartan subalgebra

$$
\mathbf{h} \subset \boldsymbol{\eta} \subset \mathbf{g} .
$$

The Killing form of Lie algera $\mathbf{g}(\boldsymbol{\eta})$ of $G(H)$ induces a canonical metric $($, on $G(H)$ and the dual space of $\mathbf{g}(\boldsymbol{\eta})$. The metric $($,$) of G$ induces a natural reductive metric $(,)_{\mathbf{m}}$ on $G / H$.

Let $\Phi_{\mathbf{g}}$ be the set of roots of $\mathbf{g}$. The roots $\Phi_{\boldsymbol{\eta}}$ of $\boldsymbol{\eta}$ form a subset of the roots of $\mathbf{g}$, i.e.,

$$
\Phi_{\eta} \subset \Phi_{\mathrm{g}} .
$$

Choosing a positive root system $\Phi_{\mathbf{g}}^{+}$for $\mathbf{g}$ also determines a positive root system $\Phi_{\eta}^{+}$for $\boldsymbol{\eta}$, where

$$
\Phi_{\eta}^{+} \subset \Phi_{\mathrm{g}}^{+} .
$$

Let $\rho_{\mathbf{g}}=\frac{1}{2} \sum_{\alpha \in \Phi_{\mathbf{g}}^{+}} \alpha$ and $\rho_{\boldsymbol{\eta}}=\frac{1}{2} \sum_{\alpha \in \Phi_{\boldsymbol{\eta}}^{+}} \alpha$ denote the Weyl vectors of $\mathbf{g}$ and $\boldsymbol{\eta}$ respectively.

Let $U_{\mu}$ be a given irreducible representation of $\boldsymbol{\eta}$ with highest weight $\mu$. Let $G \times{ }_{H} U_{\mu}$ be an associated vector bundle of the principal bundle $P(G / H, H)$. The Hilbert space of square integrable sections of $G \times{ }_{H} U_{\mu}$ decomposes into the direct sum of the eigenspaces of the Laplacian on $G / H$, which are irreducible representations $V_{\lambda}$ of $\mathbf{g}$ with highest weights $\lambda$ 's. and this induces the following expression for the Laplacian on $G / H$ which was discussed in [2], [3], [4], [5], [6], [7], [8], [9], and appears explicitly in [10].

Definition 1. The Laplacian on $G / H$ is

$$
\Delta=C_{2}(\mathbf{g}, \cdot)-C_{2}(\boldsymbol{\eta}, U) .
$$

Here $C_{2}(\mathbf{g}, \cdot)$ is the quadratic Casimir element of $\mathbf{g}$ calculated in an irreducible representation of $\mathbf{g} . C_{2}(\boldsymbol{\eta}, U)$ is the quadratic Casimir element of $\boldsymbol{\eta}$ calculated in a given irreducible representation $U$. 
Lemma 2. The value of the scalar curvature $R$ of $G / H$ is given by

$$
R=\frac{1}{4} t r_{\mathbf{m}} C_{2}=6\left[\left(\rho_{\mathbf{g}}, \rho_{\mathbf{g}}\right)-\left(\rho_{\boldsymbol{\eta}}, \rho_{\boldsymbol{\eta}}\right)\right] .
$$

Proof. Let $X_{1}, X_{2}, \cdots, X_{\text {dimm }}$ be an orthonormal basis of $\mathbf{m}$. Let $R_{m}$ be the Riemann curvature on $\mathbf{m}$.

$$
\begin{aligned}
R & =\sum_{i, j=1}^{\operatorname{dimm}} R_{m}\left(X_{i}, X_{j}, X_{j}, X_{i}\right) \\
& =\frac{1}{4} \sum_{i, j=1}^{\operatorname{dimm}}\left(\left[\left[X_{i}, X_{j}\right], X_{j}\right], X_{i}\right) \\
& =\frac{1}{4} \sum_{i, j=1}^{\operatorname{dimm}}\left(\operatorname{ad}_{X_{j}} \circ \operatorname{ad}_{X_{j}}\left(X_{i}\right), X_{i}\right) \\
& =\frac{1}{4} \sum_{i=1}^{\operatorname{dimm}}\left(\operatorname{ad}\left(C_{2}\right) X_{i}, X_{i}\right) \\
& =\frac{1}{4} \operatorname{tr}_{\mathbf{m}}\left(C_{2}\right) .
\end{aligned}
$$

By a result of Kostant [11], $\frac{1}{24} \operatorname{tr}_{\mathbf{g}}\left(C_{2}\right)=\left(\rho_{\mathbf{g}}, \rho_{\mathbf{g}}\right)$, we have

$$
\frac{1}{24} \operatorname{tr}_{\mathbf{m}}\left(C_{2}\right)=\left(\rho_{\mathbf{g}}, \rho_{\mathbf{g}}\right)-\left(\rho_{\boldsymbol{\eta}}, \rho_{\boldsymbol{\eta}}\right) .
$$

This concludes the proof of the lemma.

Let $D$ be the Dirac operator on $G / H$. In [9], Dolan gave the relation among the square of Dirac operatior $D^{2}$, the Laplacian $\Delta$ and the scalar curvature $R$ on $G / H$

$$
D^{2}=\Delta+c R,
$$

where $c$ is a constant. In [9], $c$ was taken to be $\frac{1}{8}$. Comparing the corresponding case of the Kostant type of Dirac operator [11] with ours , we adopt that

$$
c=\frac{1}{6} .
$$

It follows that the square of Dirac operator on $G / H$ is

\section{Proposition 3.}

$$
D^{2}=C_{2}(\mathbf{g}, \cdot)-C_{2}(\boldsymbol{\eta}, U)+\left(\rho_{\mathbf{g}}, \rho_{\mathbf{g}}\right)-\left(\rho_{\boldsymbol{\eta}}, \rho_{\boldsymbol{\eta}}\right) .
$$

By Proposition 2 and Theorem 4 in [1], we have the following result. 
Theorem 4. Given an irreducible representation $U_{\mu}$ of $\boldsymbol{\eta}$ with highest weight $\mu$. The eigenvalue of $D^{2}$ labelled by a highest weight $\lambda$ reads

$$
E_{\lambda}=\left(\lambda+\rho_{\mathbf{g}}, \lambda+\rho_{\mathbf{g}}\right)-\left(\mu+\rho_{\boldsymbol{\eta}}, \mu+\rho_{\boldsymbol{\eta}}\right)
$$

with

$$
\left(\lambda+\rho_{\mathbf{g}}, \lambda+\rho_{\mathbf{g}}\right) \geq\left(\mu+\rho_{\boldsymbol{\eta}}, \mu+\rho_{\boldsymbol{\eta}}\right) .
$$

The multiplicity of the eigenvalue $E_{\lambda}$ of $D^{2}$ is given by the Weyl dimension formula:

$$
\operatorname{dim} V_{\lambda}=\frac{\prod_{\alpha \in \Phi_{\mathrm{g}}^{+}}\left(\lambda+\rho_{\mathbf{g}}, \alpha\right)}{\prod_{\alpha \in \Phi_{\mathrm{g}}^{+}}\left(\rho_{\mathbf{g}}, \alpha\right)} .
$$

Moreover, If there exists an element $w \in W_{\mathbf{g}}$ in the Weyl group of $\mathbf{g}$ such that the weight $w\left(\mu+\rho_{\boldsymbol{\eta}}\right)-\rho_{\mathbf{g}}$ is dominant for $\mathbf{g}$. Then The lowest eigenvalue of $D^{2}$ is

$$
E_{w\left(\mu+\rho_{\boldsymbol{\eta}}\right)-\rho_{\mathrm{g}}}=0
$$

and the multiplicity of the lowest eigenvalue of $D^{2}$ is just the index of Dirac operator,

$$
\operatorname{Ind} D=\operatorname{dim} V_{w\left(\mu+\rho_{\boldsymbol{\eta}}\right)-\rho_{\mathrm{g}}}=\frac{\prod_{\alpha \in \Phi_{\mathrm{g}}^{+}}\left(w\left(\mu+\rho_{\boldsymbol{\eta}}\right), \alpha\right)}{\prod_{\alpha \in \Phi_{\mathrm{g}}^{+}}\left(\rho_{\mathbf{g}}, \alpha\right)} .
$$

Remark. $V_{w\left(\mu+\rho_{\boldsymbol{\eta}}\right)-\rho_{\mathrm{g}}}$ is, up to a sign, equal to the $G$-equivariant index of the Kostant's Dirac operator on $G / H$ [11], [12], [13], [14].

\section{References}

[1] L. Hu, The Laplacian on homogeneous spaces, J. Math. Phys., 49 (2008), 053513.

[2] C. N. Yang, Generalization of Dirac monopole to $S U_{2}$ gauge fields, J. Math. Phys., 19 (1978), 320-328.

[3] A. Salam and Strathdee, On Kaluza-Klein theory, Ann. Phys., 141 (1982), 316-352.

[4] M. Stone, Supersymmetry and the quantum mechanics of spin, Nucl. Phys. $B, 314$ (1989), 557-586.

[5] N. P. Landsman, Induced representations, gauge fields, and quantization on homogeneous spaces, Rev. Math. Phys., 4 (1992), 503-528. 
[6] P. Lévay, D. McMullan and I.Tsutsui, The canonical connection in quantum mechanics, J. Math. Phys., 37 (1996), 625-636.

[7] S.-C. Zhang and J. Hu, A four dimensional generalization of the quantum Hall effect, Science, 294 (2001), 823-828.

[8] D. Karabali and V. P. Nair, Quantum Hall effect in higher dimensions, Nucl. Phys. B, 641 (2002), 533-546.

[9] B. Dolan, The spectrum of the Dirac operator on coset spaces with homogeneous gauge fields, JHEP, 0305 (2003), 018.

[10] G. Meng, Geometric construction of the Quantum Hall Effect in all even dimensions, J. Phys. A, 369415 (2003), 9415-9424.

[11] B. Kostant, A cubic Dirac operator and the emergence of the Euler number multiplets of representations for equal rank subgroups, Duke Math. J., 100 (1999), 447-501.

[12] R. Bott, The index theorem for homogeneous differential operators, In: Differential and Combinatorial Topology, edited by S. S. Cairns, Princeton University Press, (1965), 167-186.

[13] B. Gross, B. Kostant, P. Ramond and S. Sternberg, The Weyl character formula, the half-spin representations, and equal rank subgroups, Proc. Natl. Acad. Sci. USA, 95 (1998), 8441-8442.

[14] G. D. Landweber, Harmonic spinors on homogeneous spaces, Representation Theory, 4 (2000), 466-473. 
